\title{
EVALUATION OF IPAD PRO 2020 LIDAR FOR ESTIMATING TREE DIAMETERS IN URBAN FOREST
}

\author{
X. Wang ${ }^{1,2, *}$, A. Singh ${ }^{3}$, Y. Pervysheva ${ }^{4}$, K. E. Lamatungga ${ }^{5}$, V. Murtinová ${ }^{6}$, M. Mukarram ${ }^{7,8}$, Q. Zhu ${ }^{1}$, K. Song ${ }^{1}$, P. Surový ${ }^{3}$, \\ M. Mokroš $2,3, *$ \\ ${ }^{1}$ School of Ecological and Environmental Sciences, East China Normal University, Shanghai 200241, China, \\ wx1332529@163.com; seed27@126.com; ksong@des.ecnu.edu.cn \\ ${ }^{2}$ Department of Forest Harvesting, Logistics and Ameliorations, Faculty of Forestry, Technical University in Zvolen, Slovakia \\ martin.mokros@tuzvo.sk; mokros@fld.czu.cz \\ ${ }^{3}$ Faculty of Forestry and Wood Sciences, Czech University of Life Sciences, Prague, Czech Republic \\ singha@fld.czu.cz; urovy@fld.czu.cz \\ ${ }^{4}$ Department of Geoinformation Technologies and Space Monitoring of the Earth, National aerospace university "Kharkiv Aviation \\ Institute", Ukraine \\ lizapervyseva@gmail.com \\ ${ }^{5}$ Department of Natural Environment, Faculty of Forestry, Technical University in Zvolen, Slovakia \\ kikiekiawan@gmail.com \\ ${ }^{6}$ Department of Applied Ecology, Faculty of ecology and environmental sciences, Technical University in Zvolen, Slovakia \\ vmurtinova@gmail.com \\ ${ }^{7}$ Advance Plant Physiology Section, Department of Botany, Aligarh Muslim University, Aligarh, India \\ mdmukarram007@gmail.com \\ ${ }^{8}$ Department of Integrated Forest and Landscape Protection, Faculty of Forestry, Technical University in Zvolen, Slovakia
}

KEY WORDS: iPad Pro 2020, LiDAR, Diameter at Breast Height, point cloud, Circle fitting, Urban Forest.

\begin{abstract}
:
Remote Sensing (RS) techniques are increasingly used in urban tree inventory measurements for their improved accuracy and promptness over the conventional methods. The focus of this study is to evaluate the application of iPad Pro 2020 and its LiDAR sensor for urban trees reconstruction and Diameter at Breast Height (DBH) measurements. Altogether, 101 trees were scanned. We have used individual- and multiple-tree scan modes with different settings (Resolution: $10 \mathrm{~mm}, 15 \mathrm{~mm}, 20 \mathrm{~mm}$; Confidence: High, Low). With these methods and settings, we have established 12 combinations. The 3DScannerAPP was used to scan and generate point clouds and to estimate DBH circle-fitting algorithm was used within the DendroCloud software. Among 12 methods, the only method with $10 \mathrm{~mm}$ resolution, high confidence, and multiple-tree mode has not achieved a $100 \%$ detection rate (97\%). For multiple-tree mode, the highest estimation accuracy was $7.52 \%$ of relative RMSE, and for single-tree mode, it was $7.27 \%$. Low confidence setting had significantly higher accuracy of DBH estimation than high confidence. Furthermore, single-tree mode had a significantly higher accuracy of DBH estimation than multiple-tree mode. The most efficient combination for DBH estimation of urban trees using 3DScannerAPP within iPad Pro 2020, when time and accuracy is considered, was multiple-tree mode with $15 \mathrm{~mm}$ resolution and low confidence.
\end{abstract}

\section{INTRODUCTION}

Urban trees provide citizens with multiple benefits, such as cooling the air temperature, purifying air from pollutants, regulating water flow, also they are improving the mental health of citizens, which makes it important to have information about such trees on an individual level, forest inventory (Mcroberts and Tomppo, 2007; Ciesielski and Sterenczak, 2019). Diameter at Breast Height $(\mathrm{DBH})$ is a critical tree parameter within urban forest inventory, due to the high correlation with height, volume, or forest biomass. Based on that it can be used to calculate multiple economic values (Nowak et al., 2002) and ecological benefits such as cooling the air temperature (Ren et al., 2013), purification air from pollution (Janhäll, 2015), and improving the mental health of citizens (Nielsen et al., 2007). Manual forest inventory is costly and time-consuming. This demands sustainable alternative methods, such as Remote Sensing (RS) methods, to derive $\mathrm{DBH}$, as well as other tree parameters (Ciesielski and Sterenczak, 2019; Shahtahmassebi et al., 2021). Terrestrial Laser Scanning (TLS) is considered to be one of the most accurate terrestrial RS methods for detailed measurements of a forest plot (Liang and Hyyppä, 2013; Liang et al., 2018). However, in some cases, the high cost and the need of specialized knowledge for TLS can be a disadvantage. To overcome this, Mobile Laser Scanner (MLS) (Liang et al., 2014) and Close-Range Photogrammetry (CRP) (Mokroš et al., 2018) were suggested as alternative RS methods. Furthermore, other novel techniques have been introduced in recent years and their feasibility to estimate DBH in forests was investigated, for example, smartphone-based Google Tango (Tomaštík et al., 2017; Hyyppä et al., 2017; Fan et al., 2018) or Microsoft Azure Kinect (Hyyppä et al., 2017; McGlade et al., 2020).

In 2020, Apple Inc. introduced a LiDAR-based depth sensor and enhanced Augmented Reality (AR) application

${ }^{*}$ Corresponding author 
programming interface (API) in the 2020 iPad Pro (799 dollars at the time of publication), which aimed to augment reality (Apple Inc., 2020). This custom-designed LiDAR Scanner works with the cameras, motion sensors, and frameworks in iPadOS to measure depth. It has a detection range of $5 \mathrm{~m}$ and operates at nanosecond speeds. Moreover, multiple corresponding APPs have been introduced after the launch of iPad Pro 2020, which made widespread use of 3D scanning possible. This combination of hardware and software makes iPad Pro 2020 a newly possible alternative RS method.

Our objective was to test the possibility of using iPad Pro 2020 to estimate the DBH of urban trees. We compared 3 different resolutions, 2 different confidence levels as well as 2 different scanning modes, which resulted in 12 methods for $\mathrm{DBH}$ estimation on urban trees $(n=101)$. We focused on: (1) the scanning possibilities of iPad Pro 2020 when urban trees are the objects of scanning; (2) the data acquisition approaches and their impact on DBH estimation accuracy; (3) the impact of different scanning settings on the accuracy of DBH estimation. With this contribution, we aimed to support future users of iPad Pro 2020 and possible of iPhone 12 Pro and Pro Max in urban forest inventory to make a well-informed decision on how to use this new RS technique, the efficiency and the limitations of this method, and which setting or scanning mode should be used for urban forest inventory.

\section{MATERIALS AND METHODS}

The object of interest for this paper was a $6423 \mathrm{~m}^{2}$ plot with 141 trees in an urban park (Figure 1) in Zvolen, Slovakia (48.57324, 19.11856). Among those trees, 101 of them were selected with DBH larger than $10 \mathrm{~cm}$. The DBH of those trees was $39.72 \mathrm{~cm}$ $\pm 19.42 \mathrm{~cm}$

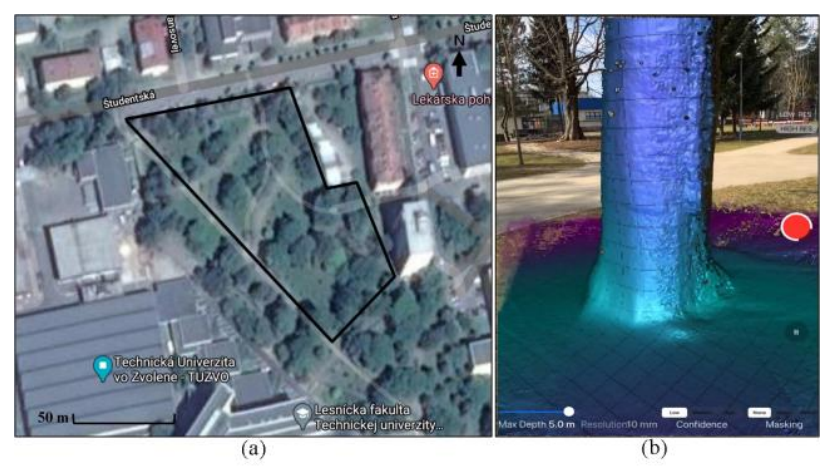

Figure 1. Urban park in Zvolen. (a) Plot area; (b) example of single-tree mode with $10 \mathrm{~mm}$ resolution at Low confidence using 3D Scanner APP in iPad Pro 2020

\subsection{Reference Data}

The positions of all trees were measured by Coordinates - GPS Formatter APP 7.1 (Mapnitude Company Limited, 2021) installed in iPad Pro 2020 at $1.3 \mathrm{~m}$ height. These positions were used to pair the reference measurements with a point cloud based measurements of DBH. In the post-processing, the pairing of positions was also based on the scanner's photos recording the tree distribution, because of 1-3 m errors from GPS in iPad Pro. The DBH of all trees was measured by a girth tape and then was recalculate to diameter. The reference data were collected by one person. It took 132 minutes.

\section{2 iPad Pro Data Collection}

We used 3DScannerAPP 1.9.1 (Laan Consulting Corp, 2021) installed in iPad Pro 2020 for scanning (Figure 1). The app provides multiple resolutions and confidence settings. The maximum range of LiDAR sensor in iPad Pro 2020 is $5 \mathrm{~m}$, which was also used in our experiment. For Masking, we chose NONE rather than OBJECT or PERSON. Confidence is an option for thresholding the data coming in from the sensor, with LOW, MEDIUM, and HIGH to choose from. For example, HIGH only keeps the best quality points but reduces the amount of data available. Resolution between $5 \mathrm{~mm}$ to $20 \mathrm{~mm}$ can be selected and a lower value $(5 \mathrm{~mm})$ means higher resolution but will increase scan size. In our experiment, we have used different resolutions: $10 \mathrm{~mm}, 15 \mathrm{~mm}$, and $20 \mathrm{~mm}$. Two confidence levels Low and High. With each setting, we have scanned the plot by multiple-tree and single-tree level mode. Table 1 shows all the used combinations.

\begin{tabular}{|c|c|c|c|}
\hline Method & Resolution $(\mathrm{mm})$ & Confidence & Scanning Mode \\
\hline 1 & 20 & High & Multiple \\
\hline 2 & 20 & Low & Multiple \\
\hline 3 & 15 & High & Multiple \\
\hline 4 & 15 & Low & Multiple \\
\hline 5 & 10 & High & Multiple \\
\hline 6 & 10 & Low & Multiple \\
\hline 7 & 20 & High & Single \\
\hline 8 & 20 & Low & Single \\
\hline 9 & 15 & High & Single \\
\hline 10 & 15 & Low & Single \\
\hline 11 & 10 & High & Single \\
\hline 12 & 10 & Low & Single \\
\hline
\end{tabular}

Table 1. Setting details.

The difference between scanning modes was that in single-tree mode each tree was scanned separately and for the multiple-tree mode the scanning was continuous. In both modes, we moved slowly "in a circle" around the trunk and the iPad Pro was perpendicular to the trunk at the breast height, at $0.5 \mathrm{~m}$ to $1 \mathrm{~m}$ distance from the trunk. The data collected with multiple-tree mode was constant. However, it was not possible to scan the whole plot at once with some settings due to the RAM limitations. Therefore, we have scanned until the application had stopped us due to the full memory. Only with method 1, we have scanned the whole plot at once. Table 2 shows different numbers of scans for all 6 different scanning methods together with the data collection and data process time.

\begin{tabular}{|c|c|c|c|}
\hline Method & $\begin{array}{c}\text { Data Collection } \\
\text { Time (min) }\end{array}$ & No. of Scans & $\begin{array}{c}\text { Data Process } \\
\text { Time (min) }\end{array}$ \\
\hline 1 & 36 & 1 & 32 \\
\hline 2 & 34 & 4 & 44 \\
\hline 3 & 34 & 4 & 49 \\
\hline 4 & 34 & 5 & 54 \\
\hline 5 & 32 & 4 & 58 \\
\hline 6 & 36 & 14 & 86 \\
\hline
\end{tabular}

Table 2. Comparison among the number of iPad Pro scans, data collection time, and data process time of the various methods for multiple-tree mode.

It took around 20 seconds for a scan by single-tree mode, except for the time of transferring to another tree (if it was included, it was 45 seconds for one tree). We explored and checked the models and exported them as point clouds within 3D Scanner APP to a laptop for further processing. 


\subsection{Estimation of Diameter at Breast Height}

A circle fitting algorithm was used to estimate DBH. The algorithm searched for diameter and the position of circle centre in 2D space on cross section of trunk at breast height $(1.3 \mathrm{~m})$. Initially, an empty grid with $0.5 \mathrm{~m} \times 0.5 \mathrm{~m}$ cell size was created. Based on the created grid, normalize $\mathrm{Z}$ values for each point of the point clouds were calculated. Then, the point cross-section was created. The cross-section consists of points with normalized $\mathrm{Z}$ values ranging from $1.28 \mathrm{~m}$ to $1.32 \mathrm{~m}$. We then spatially clustered cross-sections to group them based on the minimum number of points and the maximum distance limit. We used $10 \mathrm{~cm}$ as a maximum distance limit and 200 points as a minimum points limit. Finally, groups were visually checked using the cross-section analyst, in which the optimal circle was used as a refining method as well. A detailed explanation of the method we used can be found in (Koreň et al., 2017). The entire DBH extraction was conducted using DendroCloud 1.21 software (Koreň, 2017). Before that, vertical rotation of point clouds was carried out in CloudCompare 2.11 software (Telecom Paris Tech and Électricité de France (EDF), 2020).

The positions of trees measured by Coordinates - GPS Formatter APP were synchronized with estimates of DBH in ArcGIS for Desktop software 10.1 (Environmental Systems Research Institute (ESRI), 2012). For every tree, the estimated DBH layers were overlaid with reference measurements and paired.

\subsection{Data Evaluation}

The error of estimation was calculated as the difference between the estimated DBH and reference DBH of the trunk. The bias was calculated as the average of errors. Based on the errors, the Root Mean Square Error (RMSE) and the relative Root Mean Square Error (rRMSE) were calculated.

Paired-samples t-tests were used to test for significant differences between measured and estimated diameters by the errors.

Three-way ANOVA and Tukey post-hoc test were used to test the influence of scanning mode, confidence level, and resolution of scanning on DBH estimation accuracy (errors).

\section{RESULTS AND DISCUSSION}

Almost all methods have a $100 \%$ detection rate, except for method 5. Only 3 trees with $18.97 \mathrm{~cm}, 20.56 \mathrm{~cm}$, and $32.02 \mathrm{~cm}$ DBH were occluded from method 5 , therefore the tree detection rate was $97 \%$. We can identify these trees as trunks clearly, but we could not identify their cross-sections at $1.28-1.3 \mathrm{~m}$. Because high resolution and confidence only keep the best quality points, so some points at $1.3 \mathrm{~m}$ of individual trees were not detected. Figure 2 shows the collected iPad Pro data that point clouds were performed on. Examples of two 3D models (single-tree and multiple-tree mode) are available online (Xiaoling et al., 2021a; Xiaoling et al., 2021b). Also, all the point clouds collected within the experiment from iPad Pro 2020 together with reference data are freely available (Xiaoling et al., 2021c).

Multiple-tree scan mode needed more likely rescan than singletree scan mode. Because in the case of multiple-tree scan mode we moved from one tree to another. When we scanned new trees, in some cases trees already scanned that were nearby were rescanned and then the scans of such trees were worse. The cross-sections at $1.3 \mathrm{~m}$ of an individual tree $(\mathrm{DBH}=34.83 \mathrm{~cm})$, from method 1 to method 12 are clearly showing that scans with multiple-tree mode have incomplete cross-sections, and had some noise inside or outside of the cross-sections in the example showed in Figure 3. On the other hand, all the singletree modes (method 7 - 12) captured whole clear cross sections in this case. And to have a stable scan, it could be difficult to avoid rescan for very near trees in multiple-tree mode. However, it didn't mean multiple-tree mode reduced accuracy dramatically than single-tree mode (Figure 4 and Figure 5).
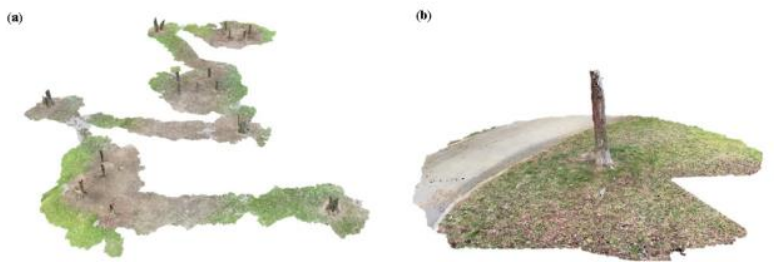

Figure 2. Demonstration of point clouds of (a) multiple-tree mode with $15 \mathrm{~mm}$ resolution and low confidence, and (b) single-tree mode with $20 \mathrm{~mm}$ resolution and low confidence.
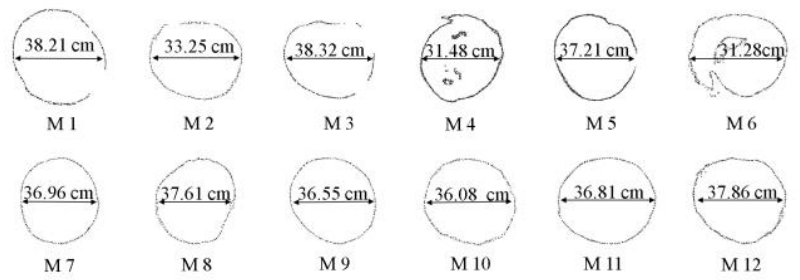

Figure 3. Illustration of the cross-section at $1.3 \mathrm{~m}$ of the same individual tree with $\mathrm{DBH}=34.38 \mathrm{~cm}$ of multiple-tree mode

(Methods 1-6) and single-tree mode (Method 7-12).

The topmost 3 accurate DBH estimations results were achieved using method $8(\mathrm{rRMSE}=7.00 \%)$, method $10(\mathrm{rRMSE}=7.27 \%)$ and method $4($ rRMSE $=7.52 \%)$; while the most inconsistent results $(13.03 \%)$ were achieved by method 5 (Table 3 ).

\begin{tabular}{|l|l|l|l|}
\hline Method & Bias $(\mathrm{cm})$ & RMSE $(\mathrm{cm})$ & rRMSE $(\%)$ \\
\hline 1 & 2.24 & 4.18 & 10.53 \\
\hline 2 & -2.45 & 4.13 & 10.40 \\
\hline 3 & 3.35 & 4.28 & 10.78 \\
\hline 4 & -0.48 & 2.99 & 7.52 \\
\hline 5 & 3.26 & 5.18 & 13.03 \\
\hline 6 & -2.36 & 4.18 & 10.52 \\
\hline 7 & 3.49 & 4.23 & 10.64 \\
\hline 8 & 1.58 & 2.78 & 7.00 \\
\hline 9 & 4.02 & 4.70 & 11.84 \\
\hline 10 & 1.57 & 2.89 & 7.27 \\
\hline 11 & 3.73 & 4.35 & 10.94 \\
\hline 12 & 1.51 & 3.21 & 8.09 \\
\hline
\end{tabular}

Table 3. The bias and RMSE resulting from the estimation of DBH.

Among multiple-tree scan mode, method 4 (Resolution $=15$ $\mathrm{mm}$, Confidence $=$ Low $)$ presented the best results $(\mathrm{rRMSE}=$ $7.52 \%$ ); on the other hand, method 5 (Resolution $=10 \mathrm{~mm}$, Confidence $=$ High) showed the most inaccurate results $($ rRMSE $=13.03 \%)($ Table 3$)$. Among single-tree mode, method 8 (Resolution $=20 \mathrm{~mm}$, Confidence $=$ Low $)$ had the optimum results $(7.00 \%)$; and method 9 (Resolution $=15 \mathrm{~mm}$, Confidence $=$ High $)$ had the poorest results $(11.84 \%)$. The most accurate DBH estimations results from multiple- and singletree scan modes had similar rRMSE (7.52\% and $7.00 \%)$. 
We also evaluated the accuracy of results by linear regression depicting the ideal relationship between the DBH estimated from iPad Pro data and reference data. As shown in Figure 4, both multiple-tree mode and single-tree mode, had the $\mathrm{R}^{2}$ above 0.96 , the highest achieved was by method 11 (Resolution $=10$ $\mathrm{mm}$, Confidence $=$ High, Scan mode $=$ Single-tree mode $)\left(\mathrm{R}^{2}=\right.$ 0.99), and method 5 (Resolution $=10 \mathrm{~mm}$, Confidence $=$ High, Scan mode $=$ Multiple-tree mode $)$ had the lowest $\mathrm{R}^{2}(0.9661)$. All single-tree modes had higher $\mathrm{R}^{2}$ than corresponding multiple-trees modes.
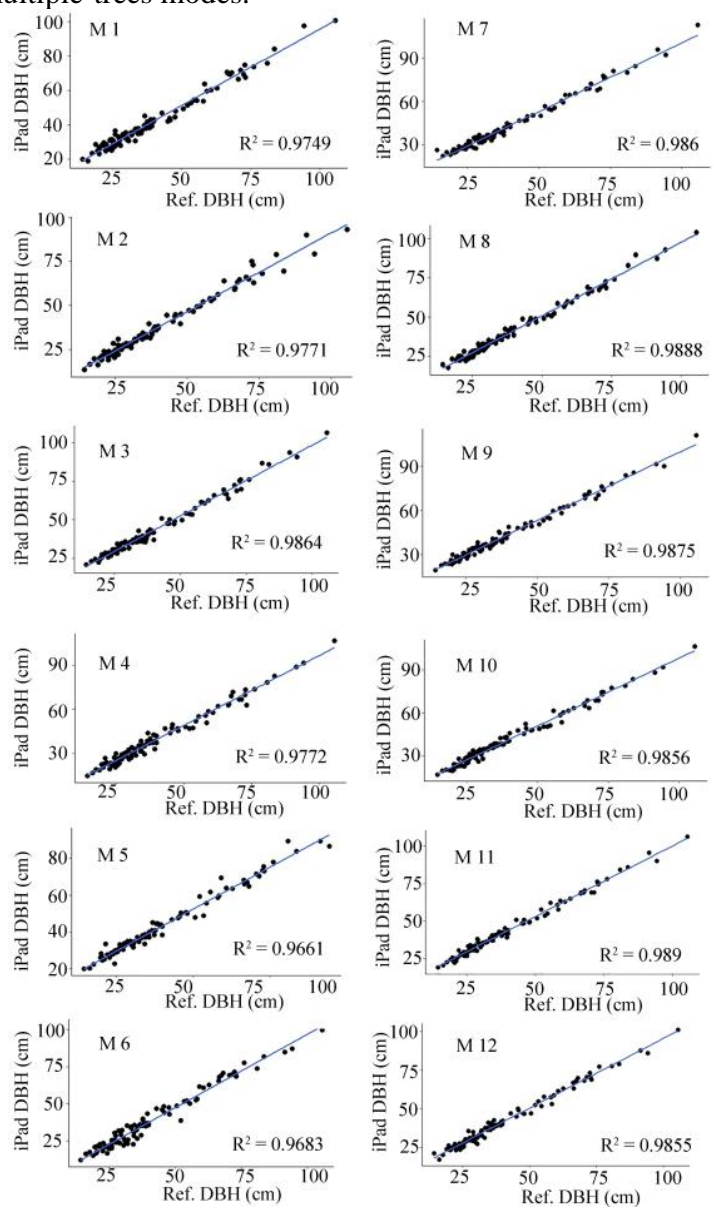

Figure 4. Scatterplot and linear regression line depicting the ideal relationship between the DBH estimated from iPad Pro data and reference data. $\mathrm{M}$ is an abbreviation of Method.

Three-way ANOVA indicated that DBH errors estimated from point cloud collected by iPad Pro data are significantly influenced by resolutions $(10 \mathrm{~mm}, 15 \mathrm{~mm}$, and $20 \mathrm{~mm})$, scanning mode (single vs. multiple), and also by confidence (low vs. high). Based on further analysis by Tukey post-hoc test we have found out that $15 \mathrm{~mm}$ resolution $($ Error $=2.08 \mathrm{~cm} \pm$ $3.18 \mathrm{~cm}$ ) is significantly different from $20 \mathrm{~mm}$ and $10 \mathrm{~mm}$ resolution (Error were $1.17 \mathrm{~cm} \pm 3.69 \mathrm{~cm}$ and $1.53 \mathrm{~cm} \pm 3.98$ $\mathrm{cm}$, respectively). It is demonstrated that iPad Pro data with low confidence $(-0.13 \mathrm{~cm} \pm 3.41 \mathrm{~cm})$ are significantly more accurate than high confidence $(3.31 \mathrm{~cm} \pm 3.01 \mathrm{~cm})$. Similarly, the singletree mode is providing higher accuracy especially when low confidence is used. The results of the three-way ANOVA are shown in Table 4.

\begin{tabular}{|c|c|c|c|c|c|}
\hline Term & df & Sum sq. & Mean sq. & Statistic & $p$-value \\
\hline Resolution & 2 & 165.23 & 82.61 & 9.47 & 0.00 \\
\hline
\end{tabular}

\begin{tabular}{|c|c|c|c|c|c|}
\hline Confidence & 1 & 3481.04 & 3481.04 & 398.83 & 0.00 \\
\hline Scan_Mode & 1 & 1231.99 & 1231.99 & 141.15 & 0.00 \\
\hline Res:Conf & 2 & 32.95 & 16.47 & 1.89 & 0.15 \\
\hline Res:Mode & 2 & 85.47 & 42.74 & 4.90 & 0.01 \\
\hline Conf:Mode & 1 & 433.15 & 433.15 & 49.63 & 0.00 \\
\hline $\begin{array}{c}\text { Res: Conf: } \\
\text { Mode }\end{array}$ & 2 & 46.24 & 23.12 & 2.65 & 0.07 \\
\hline Residuals & 1164 & 10159.4 & 8.73 & NA & NA \\
\hline
\end{tabular}

Table 4. Results of three-way ANOVA

A boxplot of DBH errors grouped by resolution, confidence, and scanning mode is shown in Figure 5. Method 4 (Scanning mode $=$ Multiple-tree mode, Resolution $=15 \mathrm{~mm}$, Confidence $=$ Low) is significantly different from all other methods. Furthermore, the bias of method 4 is the only one that is not significantly different from 0 , and at the same time, it is the most accurate multi-tree scanning mode. Method 6 and method 2 significantly underestimated the reference DBH. All methods with single-tree mode are significantly overestimated reference DBH. Also, methods 1, 3, and 5 are significantly overestimating reference DBH. We have used a t-test.

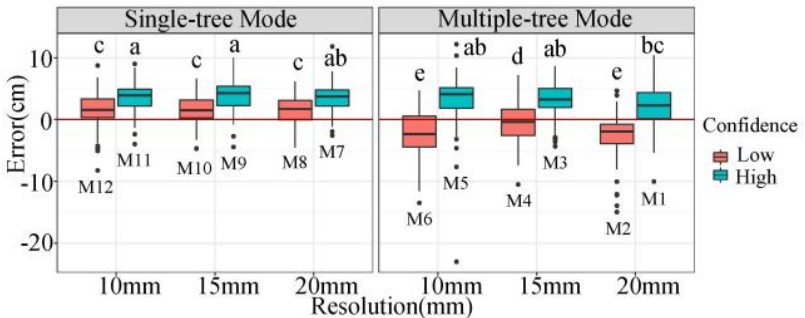

Figure 5. Boxplot for estimating DBH error using iPad Pro with different settings. Significant difference analysis among 12 methods were shown in the graph. Letter a, ab, bc, c, d, and e presented the significance levels (if two variables have different letters, they are significantly different). $\mathrm{M}$ is an abbreviation of Method.

The average time needed to collect the data from single-tree mode (methods 7-12) was 75 minutes (approximately 45 seconds for a single tree with saving and moving to another tree) The time needed for multiple-tree mode (methods 1-6) ranged from 32 minutes to 36 minutes. The time needed to collect reference data was 132 minutes. On the other hand, the time needed to process the data from the single-tree method was around 200 minutes. The time is higher due to the used software where we had to process each tree separately and manually import the data and go through the workflow manually with each of the single trees. Therefore, the time can be reduced dramatically with fully automatic data processing. However, multiple-tree mode methods (method 1-6) took less time due to the lower number of scans. The fastest was method 1 where only one scan was needed for the whole plot (32 minutes) and the slowest was method 6 where 14 scans were needed (86 minutes).

\section{CONCLUSIONS}

This experiment aimed to evaluate the possibility of $\mathrm{DBH}$ estimation from point cloud produced by iPad Pro 2020 in an urban forest. For beginners in this field, we intended to provide a guide in choosing setup and workflow to be adapted for diameter extraction of trees situated in an urban park. 
For all 12 methods with all their different resolution, confidence, and scanning mode, individual tree DBH could be calculated successfully from iPad Pro point clouds. Almost all methods have a $100 \%$ detection rate. Only method 5 (Resolution $=10$ $\mathrm{mm}$, Confidence $=$ High, Scan mode $=$ Multiple-tree mode) occluded 3 trees at cross-section step, because of high resolution and confidence it only kept the best quality points, but still had a $97 \%$ detection rate.

All 12 methods had acceptable estimation of DBH. For multiple-tree mode, the best result was from method 4 $($ Resolution $=15 \mathrm{~mm}$, Confidence $=$ Low $)$ with $7.52 \%$ rRMSE, and for single trees scan, it was method 8 (Resolution $=20 \mathrm{~mm}$, Confidence $=$ Low $)$ with $7.00 \%$ rRMSE.

iPad Pro data with low confidence $(-0.13 \mathrm{~cm} \pm 3.41 \mathrm{~cm})$ can significantly contribute to better accuracy when compared to high confidence $(3.31 \mathrm{~cm} \pm 3.01 \mathrm{~cm})$. Similarly, the single-tree mode is providing higher accuracy especially when low confidence is used. But we recommend multiple-tree mode, especially method $4($ Resolution $=15 \mathrm{~mm}$, Confidence $=$ Low $)$ which had high accuracy $($ RMSE $=2.99 \mathrm{~cm}$, Bias $=-0.48 \mathrm{~cm}$, rRMSE $=7.52 \%, \mathrm{R}^{2}=0.9749$, error $=-0.48 \mathrm{~cm} \pm 2.96 \mathrm{~cm}$ ) because it took lesser time in the field and office and present the best result among methods with multiple-tree mode.

Method 6 (Scanning mode $=$ Multiple-tree mode, Resolution $=$ $10 \mathrm{~mm}$, Confidence $=$ Low $)$ and method $2($ Scanning mode $=$ Single-tree mode, Resolution $=10 \mathrm{~mm}$, Confidence $=$ Low $)$ significantly underestimated the reference DBH. Only method method 4 had not significantly over- or underestimated the reference DBH. Conclusively, we suggested that, the iPad Pro 2020 with 3D Scanner APP 1.9.1 (Maximum depth/ Range $=5$ $\mathrm{m}$, Masking $=$ None, Resolution $=15 \mathrm{~mm}$, Confidence $=$ Low with multiple-tree mode could provide a feasible, sufficiently accurate, and cost-effective solution for DBH measurements in urban forests.

\section{ACKNOWLEDGEMENTS}

This research was funded by grant No. CZ.02.1.01/0.0/0.0/16_019/0000803 (“Advanced research supporting the forestry and wood-processing sector's adaptation to global change and the 4th industrial revolution") financed by OP RDE and by Scientific Grant Agency (VEGA) of the Ministry of Education, Science, Research and Sport of the Slovak Republic and the Slovak Academy of Sciences grant number1/0335/20 (A multi-camera system prototype as a tool for creating a highly detailed model of individual trees and forest stands)

\section{REFERENCES}

Apple Inc., 2020. iPad Pro 11, Version 14.6. Available online: https://www.apple.com/newsroom/2020/03/apple-unveils-newipad-pro-with-lidar-scanner-and-trackpad-support-in-ipados/ (3 March 2020)

Ciesielski, M. and Sterenczak, K., 2019. Accuracy of determining specific parameters of the urban forest using remote sensing, IForest (Viterbo), 12, 498-510, 10.3832/ifor3024-012.
Environmental Systems Research Institute (ESRI), 2012. ArcGIS for Desktop software, Version 10.1. Available online: https://support.esri.com/en/download/1913 (6 June 2012)

Fan, Y., Feng, Z., Mannan, A., Khan, T., Shen, C., and Saeed, S., 2018. Estimating Tree Position, Diameter at Breast Height, and Tree Height in Real-Time Using a Mobile Phone with RGB-D SLAM, Remote sensing (Basel, Switzerland), 10, 1845, $10.3390 / \mathrm{rs} 10111845$.

Hyyppä, J., Virtanen, J.-P., Jaakkola, A., Yu, X., Hyyppä, H., and Liang, X., 2017. Feasibility of Google Tango and Kinect for Crowdsourcing Forestry Information, Forests, 9, 6, 10.3390/f9010006.

Janhäll, S., 2015. Review on urban vegetation and particle air pollution - Deposition and dispersion, Atmos. Environ., 105, 130-137, 10.1016/j.atmosenv.2015.01.052.

Koreň, M., Mokroš, M., and Bucha, T., 2017. Accuracy of tree diameter estimation from terrestrial laser scanning by circlefitting methods, International Journal of Applied Earth Observation and Geoinformation, 63, 122-128, 10.1016/j.jag.2017.07.015.

Koreň, M., 2017. DendroCloud: Point Cloud Processing Software for Forestry, Version 1.45. Available online: http://gis.tuzvo.sk/dendrocloud/download/dendrocloud_1_45.pd f (30 January 2018).

Laan Consulting Corp, 2021. 3D Scanner APP, Version 1.9.1. Available online: https://apps.apple.com/us/app/3d-scannerapp/id1419913995 (1 March 2021).

Liang, X. and Hyyppä, J., 2013. Automatic stem mapping by merging several terrestrial laser scans at the feature and decision levels, Sensors (Switzerland), 13, 1614-1634, 10.3390/s130201614.

Liang, X., Kukko, A., Kaartinen, H., Hyyppä, J., Yu, X., Jaakkola, A., and Wang, Y., 2014. Possibilities of a Personal Laser Scanning System for Forest Mapping and Ecosystem Services, Sensors, 14, 10.3390/s140101228.

Liang, X., Hyyppä, J., Kaartinen, H., Lehtomäki, M., Pyörälä, J., Pfeifer, N., Holopainen, M., Brolly, G., Francesco, P., Hackenberg, J., Huang, H., Jo, H.-W., Katoh, M., Liu, L., Mokroš, M., Morel, J., Olofsson, K., Poveda-Lopez, J., Trochta, J., Wang, D., Wang, J., Xi, Z., Yang, B., Zheng, G., Kankare, V., Luoma, V., Yu, X., Chen, L., Vastaranta, M., Saarinen, N., and Wang, Y., 2018. International benchmarking of terrestrial laser scanning approaches for forest inventories, ISPRS Journal of Photogrammetry and Remote Sensing, 144, 137-179, 10.1016/j.isprsjprs.2018.06.021.

McGlade, J., Wallace, L., Hally, B., White, A., Reinke, K., and Jones, S., 2020. An early exploration of the use of the Microsoft Azure Kinect for estimation of urban tree Diameter at Breast Height, Remote sensing letters, 11, 963-972, 10.1080/2150704X.2020.1802528.

McRoberts, R. E., and Tomppo, E. O., 2007. Remote sensing support for national forest inventories, Remote Sens. Environ., $110,412-419$.

Mapnitude Company Limited, 2021. Coordinates - GPS Formatter APP, Version 7.1. Available online: 
https://apps.apple.com/us/app/coordinates-gps formatter/id494286614 (15 March 2021).

Mokroš, M., Liang, X., Surový, P., Valent, P., Čerňava, J., Chudý, F., Tunák, D., Saloň, I., and Merganič, J., 2018. Evaluation of close-Range photogrammetry image collection methods for estimating tree diameters, ISPRS International Journal of Geo-Information, 7, 93, 10.3390/ijgi7030093.

Nielsen, A. B., Olsen, S. B., and Lundhede, T., 2007. An economic valuation of the recreational benefits associated with nature-based forest management practices, Landscape Urban Plann., 80, 63-71

Nowak, D. J., Crane, D. E., and Dwyer, J. F., 2002. Compensatory value of urban trees in the United States, Journal of Arboriculture, 28, 194-199

Ren, Z., He, X., Zheng, H., Zhang, D., Yu, X., Shen, G., and Guo, R., 2013. Estimation of the relationship between urban park characteristics and park cool island intensity by remote sensing data and field measurement, Forests, 4, 868-886, 10.3390/f4040868.

Shahtahmassebi, A. R., Li, C., Fan, Y., Wu, Y., lin, Y., Gan, M., Wang, K., Malik, A., and Blackburn, G. A., 2021. Remote sensing of urban green spaces: A review, Urban For. Urban Gree., 57, 126946, https://doi.org/10.1016/j.ufug.2020.126946.

Telecom Paris Tech and Électricité de France (EDF), 2020. CloudCompare software, Version 2.11. Available online: http://cloudcompare.org/release/index.html (29 July 2020)

Tomaštík, J., Saloň, Š., Tunák, D., Chudý, F., and Kardoš, M., 2017. Tango in forests - An initial experience of the use of the new Google technology in connection with forest inventory tasks, Comput. Electron. Agric., 141, 109-117, 10.1016/j.compag.2017.07.015.

Xiaoling W., Arunima S., Yelyzaveta P., Kiki Ekiawan L., Veronika M., Mohammad M., Qichong Z., Kun S., Martin M., 2021a. Multiple-tree mode example. Available online: https://sketchfab.com/3d-models/multiple-tree-mode-example88ec43e23e204b4297827ce751e48d4f

Xiaoling W., Arunima S., Yelyzaveta P., Kiki Ekiawan L., Veronika M., Mohammad M., Qichong Z., Kun S., Martin M., 2021b. Multiple-tree mode example. Available online: https://sketchfab.com/3d-models/single-tree-mode-example7b23b391d23c4b26abce1874ca92f10c.

Xiaoling W., Arunima S., Yelyzaveta P., Kiki Ekiawan L., Veronika M., Mohammad M., Qichong Z., Kun S., Martin M., 2021c. Urban Trees Inventory Data with Tape and iPad Pro 2020 Laser Scanner. Mendeley Data, Version 1, 10.17632/d9vmn6pfhj.1. 